\title{
Surface modification to improve in vitro attachment and proliferation of human urinary tract cells
}

\author{
R. MARCOVICH, B. SEIFMAN, R. BEDUSCHI and J.S. WOLF Jr \\ Urology Service, Department of Veterans Affairs Medical Center, and Department of Urology, University of Michigan Health System, Ann Arbor, MI, \\ USA
}

Accepted for publication 29 April 2003

\section{OBJECTIVE}

To evaluate the attachment and proliferation of cultured human urinary tract cells to culture plates surface-modified by photochemical immobilization of extracellular matrix (ECM) proteins.

\section{MATERIALS AND METHODS}

Human uroepithelial (UEC) and smooth muscle (SMC) cells were harvested from ureter and expanded in culture; 24-well culture plates surface-modified by photochemical covalent immobilization of ECM proteins were then seeded with UEC or SMC. To characterize cellular attachment, cells were incubated on surface-modified plates for 30 and $90 \mathrm{~min}$. For proliferation assays the cells were incubated for 3-12 days. Standard tissue culture plates with no surface modification and sham-modified plates served as controls. Differential attachment and proliferation on the various surfaces were assessed using analysis of variance with Fisher's posthoc test for multiple comparisons.

\section{RESULTS}

Attachment at 30 and 90 min of both UEC and SMC on plates surface-modified with ECM proteins was significantly greater than in control plates. Surface-modification with collagen resulted in significantly greater cellular attachment than with either laminin or fibronectin. UEC proliferation was also significantly greater than in control plates by surface-modification with collagen and fibronectin, but not with laminin. SMC proliferation was significantly better after surface modification than on sham- modified plates, but was no better than standard plates.

\section{CONCLUSIONS}

Covalent photochemical immobilization of ECM proteins to potential growth surfaces enhances the attachment of cultured UEC and SMC and the proliferation of UEC. This technique might be useful in modifying surface properties of synthetic polymer-based materials in a controlled and defined manner, giving them the capacity to promote and sustain the growth of urinary tract cells. This may lead to development of alternative methods of tissue engineering in the urinary tract.

\section{KEYWORDS}

tissue engineering, cell culture, extracellular matrix, cell adhesion, proliferation

\section{INTRODUCTION}

Finding an optimal material for urinary tract reconstruction has long been a goal in urology. For years, intestinal segments have been the major source of tissue for clinical augmentation or replacement of the urinary bladder, but their use can be problematic because of well-documented associated complications [1]. Various experimental approaches to the problem have been attempted, using both synthetic and biological materials [2]. Recent investigations in animal models have focused on biodegradable substrates, e.g. small intestinal submucosa (SIS) [3] and polyglycolic acid (PGA) polymers $[4,5]$. These materials have shown promise, but have not yet entered the clinical arena.

For a material to integrate successfully into the urinary tract it must provide native or transplanted cells with an effective growth environment. It must promote cellular growth and differentiation in an architecturally normal pattern, to preserve both the transport and barrier functions of the urinary tract. Since the introduction of new biomaterials for urinary reconstruction is likely, a method for endowing these materials with the ability to promote cellular growth might be of benefit.

One potential technique to accomplish this involves a process known as photochemical covalent immobilization [6]. In this process, extracellular matrix (ECM) proteins, e.g. collagen or fibronectin, can be covalently bonded to virtually any synthetic polymer, essentially resurfacing the material, so that there is a more hospitable environment for cellular growth. Figure 1 shows a schematic diagram of the technique, developed by SurModics, Inc. (Eden Prairie, MN, USA). The ECM molecule is linked to an aryl azide or aryl ketone compound (A), which is subsequently photoactivated by ultraviolet irradiation. An unstable, reactive intermediate (ECM-A:) is generated and covalently bonds to side groups of the target polymer, thus permanently linking the molecule to the polymer surface.

Photochemical coupling provides a surface coating superior to that obtained by simple adsorption of molecules to polymer surfaces, as shown by studies on various cell lines, including endothelial cells, fibroblasts, epithelial cells, and osteoblasts [6]. The technique has been used to alter the surface properties of vascular grafts to promote endothelialization. Clapper et al. [7] showed that when ECM proteins were covalently photocoupled to expanded PTFE or polyurethane vascular grafts, in vitro endothelial cell growth was 10-20 times greater than on uncoated vascular grafts or 
FIG. 1. Schematic diagram of the photochemical immobilization process (hv =irradiation).
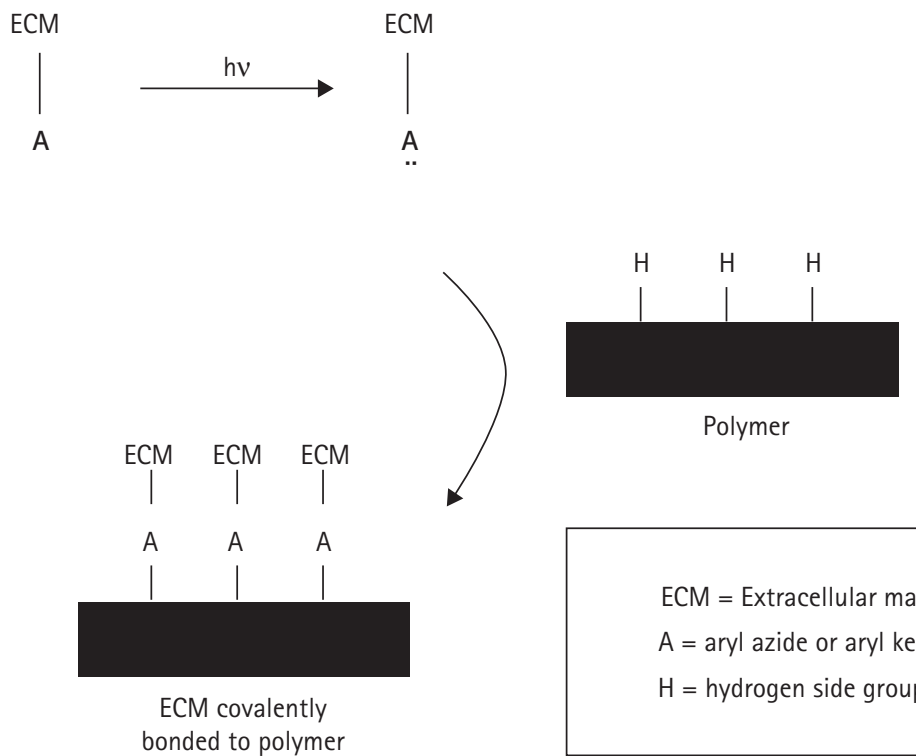

Polymer bonded to polymer

grafts coated with ECM by adsorption alone. Similarly, expanded PTFE femoral artery grafts photoimmobilized with collagen IV and fibronectin resulted in higher patency rates and significantly better luminal coverage in an in vivo canine model than did uncoated grafts.

The effect of photochemical immobilization of ECM proteins to potential synthetic growth surfaces has not been studied with cells from the urinary tract. Therefore, we sought to evaluate the attachment and proliferation of human urinary tract cells on plates surfacemodified by photochemical coupling with ECM proteins, as proof-of-principle that this technique has potential for use in urinary reconstruction.

\section{MATERIALS AND METHODS}

Segments $(1-2 \mathrm{~cm})$ of distal ureter were obtained from healthy human renal donors. Uroepithelial (UEC) and urinary tract smooth muscle (SMC) cells were harvested from these and separately expanded in cell culture as previously described [8]. UEC and SMC were grown in keratinocyte serum-free medium (KSFM) and medium 199 with 20\% fetal bovine serum (FBS; Invitrogen, Grand Island, NY, USA), respectively. At near-confluence, cells were dissociated with trypsin and passaged. Cells were used in experimental assays at the fourth passage.
As the purpose of these experiments was 'proof of principle', we chose to use tissueculture plates for all attachment and proliferation assays; 24-well polystyrene plates were obtained from SurModics on a work-for-hire basis. Thus, in these experiments, the polymer indicated in Fig. 1 was polystyrene. Wells in each row were surface-modified by the photochemical immobilization technique with either collagen IV (row 1), laminin (row 2), or fibronectin (row 3). Row 4 was left uncoated but was photoirradiated like the other rows, and served to control for any confounding from the irradiation process. Two other controls were used in all attachment and proliferation assays: a standard, commercially available plate of tissue-culture polystyrene (Becton Dickinson, Franklin Lakes, NJ, USA) and an uncoated, unirradiated polystyrene plate. Commercially available tissue-culture plates are constructed of modified polystyrene with ionized side groups, to facilitate cellular attachment. Therefore, the standard tissue culture plate was used as a control to gauge the response of cells grown on the experimental surfaces relative to their behaviour in normal tissue culture conditions. The uncoated, unirradiated plates were necessary to assess the complete effect of the experimental ECM proteins compared with the effect of unadulterated polystyrene. We chose not to include plates with adsorbed ECM as a fourth control, as in other systems covalent attachment of ECM has been shown to be superior to adsorbed ECM $[6,7]$. For all experiments 12 plates each were used for collagen, laminin, fibronectin, uncoated/ irradiated, uncoated/unirradiated, and tissue culture polystyrene (TC-P).

Adhesion assays. UEC and SMC were assessed separately; UEC were seeded on the six different surfaces at either 20000 or 40000 cells $/ \mathrm{cm}^{2}$ in $1 \mathrm{~mL} \mathrm{KSFM} \mathrm{per} \mathrm{well} \mathrm{and} \mathrm{placed} \mathrm{in}$ a humidified incubator at $37^{\circ} \mathrm{C}$ with $5 \% \mathrm{CO}_{2}$. KSFM was supplied by the manufacturer with supplemental epidermal growth factor and bovine pituitary extract to enhance cell growth. Neither of these factors was used in the media during expansion of the harvested cells, nor during any of the assays, to prevent confounding by potential growth-enhancing effects of these two agents.

Assays were carried at both 30 and $90 \mathrm{~min}$; at the designated time, free cells were rinsed off with three gentle washes of $37^{\circ} \mathrm{C}$ PBS. The remaining cells were detached by trypsinization and the contents of each well transferred to $5 \mathrm{~mL}$ tubes and then sonicated to release their DNA content. An aliquot from each tube was suspended in PBS, bis-benzimide $0.2 \mu \mathrm{g} / \mathrm{mL}$ (Sigma-Aldrich, St. Louis, MO, USA) added, and the DNA concentration of each well determined in duplicate by a fluorescence assay using an FL600 fluorescence/absorbance microplate reader at 360/460 $\mathrm{nm}$ (Bio-Tek Instruments, Winooski, VT, USA). The concentration of DNA was used as an indicator of the quantity of cells that had remained adherent in each well of the 24-well plate [9] (DNA content is an accepted surrogate for cell number [10-13]). As the focus of the investigation was the relative amount of cells on the various materials, it was not deemed necessary to calculate the absolute cell number.

The attachment of SMC was analysed analogously, with 40000 cells $/ \mathrm{cm}^{2}$ seeded and sampled at 30 and $90 \mathrm{~min}$. These assays were carried out in $1 \mathrm{~mL}$ medium-199 per well with no FBS, to exclude effects of undefined factors in the latter.

Proliferation assays. UEC and SMC were again studied separately; UEC were seeded on the six different surfaces in $1 \mathrm{~mL} \mathrm{KSFM}$ (no additives) per well at a density of 12500 cells $/ \mathrm{cm}^{2}$. Cells were maintained in culture for 5 days and the media changed on day 3. Growth was not allowed to reach confluence, to prevent confounding from 
cells possibly reaching maximum density in the culture well. Before trypsinization, free cells were removed with a PBS rinse, and afterward the contents of each well were sonicated, and DNA assayed as above to quantify the cells in each well. SMC proliferation was assessed analogously; for these assays, it was necessary to add FBS to the medium for any cellular growth to occur. Various seeding densities and concentrations of FBS were investigated. Cells were allowed to grow for 3 or 12 days. The cell content in each well was quantified as above with the DNA fluorescence assay.

Biosynthesis assay. To assess whether UEC grown on surface-modified plates retained their biosynthetic capacity, they were assayed for the production of glycosaminoglycans (GAGs), which are known to be synthesized by UEC, and which may contribute to the lack of permeability to urine of the bladder lining [14]. UEC were plated in $1 \mathrm{~mL} \mathrm{KSFM} \mathrm{in} \mathrm{24-well}$ plates photoimmobilized with collagen IV, laminin, and fibronectin. The medium was changed every 2-3 days until cells were confluent; 3 days after confluence the conditioned medium was removed and assayed for the presence of sulphated GAG, using a commercially available dye-binding assay (Blyscan Assay, Biocolor Ltd, Belfast, UK). This assay is based on specific binding of 1,9-dimethylmethylene blue to sulphated GAG [15]; $10 \mu \mathrm{L}$ of conditioned medium was combined with $1 \mathrm{~mL}$ dye reagent and mixed at room temperature for $30 \mathrm{~min}$. Samples were then centrifuged for $10 \mathrm{~min}$ and the precipitated GAG-dye complex dissolved in $1 \mathrm{~mL}$ dissociation reagent. Absorbance of bound dye was measured at $656 \mathrm{~nm}$. Plain KSFM incubated with no cells in the photoimmobilized plates was assayed to verify that there was no spurious GAG activity.

Differences in mean DNA concentration in the attachment and proliferation assays were determined by ANOVA, using Fisher's posthoc test for multiple comparisons, and statistical significance set at $P<0.05$.

\section{RESULTS}

Table 1 summarizes the attachment at 30 and 90 min of UEC expressed as the mean DNA concentration. At $30 \mathrm{~min}$, plates photoimmobilized with collagen and laminin had significantly higher rates of UEC

TABLE 1 The assessments of UEC and SMC attachment at 30 and 90 min, and UEC proliferation, for the various treatments

\begin{tabular}{lcccccc} 
Mean (SD) DNA, & Substrate & & & \\
\cline { 2 - 7 }$\mu$ /well & Collagen & Laminin & Fibronectin & TC-P & UI & UU \\
\hline Attachment, min & & & & & & \\
UEC 30 & $647(395)$ & $605(143)$ & $293(218)$ & $470(176)$ & $171(186)$ & $113(108)$ \\
UEC 90 & $1103(243)$ & $857(186)$ & $650(326)$ & $679(255)$ & $68(47)$ & $112(79)$ \\
SMC 30 & $804(69)$ & $455(94)$ & $232(68)$ & $62(38)$ & 0 & 0 \\
SMC 90 & $1251(243)$ & $1121(231)$ & $1103(189)$ & $725(203)$ & 0 & 0 \\
Proliferation & & & & & & \\
UEC at 5 days & $935(89)$ & $497(122)$ & $547(85)$ & $382(127)$ & $439(65)$ & $419(83)$
\end{tabular}

UI, uncoated, irradiated; UU, uncoated, unirradiated. For UEC at $30 \mathrm{~min}, \mathrm{P}<0.01$ for collagen vs fibronectin and $\mathrm{UI} / \mathrm{UU}$, and for laminin vs fibronectin and $\mathrm{UI} / \mathrm{UU}$, and $\mathrm{P}=0.06$ for collagen vs TC-P. For UEC at $90 \mathrm{~min}, \mathrm{P}<0.01$ for collagen vs all others and laminin vs all except TC-P $(=0.05)$, fibronectin vs UI/UU and TC-P vs UI/UU. For SMC at $30 \mathrm{~min}, \mathrm{P}<0.01$ for each of collagen, laminin, fibronectin and TC-P vs all. At $90 \mathrm{~min} P<0.05$ for collagen vs all, laminin vs all except fibronectin, fibronectin vs all except laminin and TC- $P$ vs all. For UEC proliferation $\mathrm{P}<0.01$ for collagen vs all, laminin vs collagen and TC-P and fibronectin vs all except laminin.

attachment than the uncoated plates $(P<0.001)$. There was a trend for collagen to give better attachment than TC-P $(P=0.06)$. By $90 \mathrm{~min}$, both collagen and laminin were significantly better than all three controls. In contrast, fibronectin provided no advantage over TC-P at either time. These results are for initial seeding densities of 40000 cells $/ \mathrm{cm}^{2}$; similar data were generated with seeding densities of 20000 cells $/ \mathrm{cm}^{2}$.

Table 1 also summarizes SMC attachment at 30 and $90 \mathrm{~min}$; interestingly there was no measurable attachment of SMC at either time on any of the uncoated controls, and it was also poor on commercially available TC-P. Collagen outperformed the other two ECM proteins, and all three were significantly better than controls $(P<0.001)$.

The proliferation of UEC (Table 1) was significantly enhanced by photoimmobilization of collagen over all other ECM proteins and controls $(P<0.001)$. Fibronectin performed better in this assay than would have been expected from the UEC attachment assay, being significantly better than TC-P $(P<0.001)$ and uncoated controls $(P<0.01)$. For SMC proliferation there was no detectable advantage of any of the ECM proteins over TC-P, which gave similar results at various cell seeding densities, proliferation times and concentrations of FBS (data not shown). There was no SMC growth under any condition on the uncoated controls.
The biosynthetic capability of the UEC was maintained when grown to confluence on the three ECM-modified surfaces, as determined by the GAG assay. Media conditioned for 3 days by confluent cells gave qualitatively equivalent amounts of GAG for all three ECM protein surfaces. No GAG was detected in plain incubated KSFM. Quantitative analysis was not possible, as all three ECM coatings yielded products at the lower limit of assay sensitivity.

\section{DISCUSSION}

Despite years of continuing effort to introduce new materials to replace or reconstruct urinary tract segments, autologous bowel remains the only currently clinically acceptable option [2]. The most promising materials tested in animals to date are porcine SIS $[3,16]$ and biodegradable PGA polymer scaffolds seeded with autologous cells $[4,5,17,18]$.

Augmentation of canine urinary bladders with SIS, a method relying on the in-growth of cells from surrounding tissue, has resulted in the regeneration of trilaminar bladder wall with re-innervation, contractility and compliance similar to that of the native bladder [16]. What makes SIS theoretically desirable as a platform for tissue regeneration is that, among other properties, it is 
composed mainly of collagen and is known to contain various growth factors, e.g. fibroblast growth factor-2, TGF- $\beta$-like growth factor and vascular endothelial growth factor $[19,20]$. On the negative side, smooth muscle organization in SIS-regenerated bladder is haphazard and the smooth muscle-toconnective tissue ratio is significantly lower than in native bladder wall [3]. Disadvantages of SIS which may hamper its introduction into clinical practice include the potential for late graft contraction and variability in efficacy among commercial lots.

Atala [21] proposed that cell transplantation, rather than interposition of a patch or graft with subsequent in-growth of native cells, is necessary to create functional urological tissues. PGA polymer scaffolds have been used successfully as delivery vehicles for autologous urinary tract cells cultured and expanded in vitro and then implanted in vivo. Using this method, PGA polymer scaffolds were used to regenerate the urinary bladder in dogs after supratrigonal cystectomy [18]. In that study, bladders reconstructed from PGA polymers surfaced with cultured urinary tract cells before implantation were histologically and functionally similar to native bladders, compared with bladders reconstructed with PGA polymers with no cell seeding, which showed loss of capacity, decreased compliance, and significant fibrosis.

In the present study we investigated the effect of modifying the surface properties of polystyrene vessels by covalent photochemical immobilization of extracellular matrix proteins. This method could serve to enhance the biocompatibility of future synthetic materials introduced for urinary reconstruction, either for patch-grafting and in-growth of neighbouring cells (as in the SIS experiments), or for transplanting cells first cultured and expanded in vitro. The technique has the potential advantage of being applicable to any synthetic polymer. Thus, a polymer judged suitable for use in reconstruction based, e.g. on its mechanical profile or time to reabsorption, or some other combination of properties, could be surfacemodified with one or more ECM proteins to promote its ability to sustain cell growth and differentiation. The growth-enhancing characteristics of the material could potentially be further augmented by photocoupling combinations of growth factors to it. The goal of this work would be to create a mass-produced, standardized material for urinary reconstruction that could be used 'off-the-shelf', thus obviating the variability inherent in a biological material such as SIS, and possibly reducing the need for in vitro autologous cell culturing techniques.

In the present study UEC and SMC attachment were enhanced by surface modification, particularly with collagen and laminin, as was the proliferation of UEC. SMC proliferation was no better than on the standard tissue culture surface, but was significantly better on than plain polystyrene. It is possible that enhanced SMC proliferation could not be detected because of methodological problems in growing these cells. SMC will not proliferate in vitro in the absence of serum, and we could not find a concentration of FBS that would allow for differential growth. Another potential reason for the lack of a difference between surface-modified and TC-P plates is that SMC synthesise collagen [22], and it is conceivable that once the cells plated on TC-P became adequately attached, they may have provided their own collagen matrix.

In conclusion, covalent photoimmobilization of ECM proteins to polystyrene plates enhances the attachment of UEC and SMC, and the proliferation of UEC. This technique may be useful for modifying the surface properties of synthetic polymer-based materials in a controlled and defined manner, endowing them with the capacity to promote and sustain the growth of urinary tract cells.

\section{ACKNOWLEDGEMENTS}

Supported by a Merit Review Grant from the United States

Department of Veterans Affairs

\section{REFERENCES}

1 McDougal WS. Metabolic complications of urinary intestinal diversion. J Urol 1992; 147: 1199-208

2 Elbahnasy AM, Shalhav A, Hoenig DM, Figenshau R, Clayman RV. Bladder wall substitution with synthetic and nonintestinal organic materials. J Urol 1998; 159: $628-37$
3 Kropp BP, Rippy MK, Badylak SK et al. Regenerative urinary bladder augmentation using small intestinal submucosa. urodynamic and histopathologic assessment in long-term canine bladder augmentations. J Urol 1996; 155: 2098-104

4 Atala A, Vacanti JP, Peters CA, Mandell J, Retik $A B$, Freeman MR. Formation of urothelial structures in vivo from dissociated cells attached to biodegradable polymer scaffolds in vitro. J Urol 1992; 148: 658-62

5 Atala A, Freeman MR, Vacanti JP, Shepard J, Retik AB. Implantation in vivo and retrieval of artificial structures consisting of rabbit and human urothelium and human bladder muscle. J Urol 1993; 150: 608-12

6 Amos RA, Anderson AB, Clapper DL et al. Biomaterial surface modification using photochemical coupling technology. In Wise DL ed. Encyclopedic Handbook of Biomaterials and Bioengineering. New York: Marcel Dekker Inc, 1995: 895-925

7 Clapper DL, Hagen KM, Hupfer JM, Anderson AB, Guire PE. Covalently immobilized ECM proteins improve patency and endothelialization of $4 \mathrm{~mm}$ grafts implanted in dogs. Trans Soc Biomat 1993; 16: 42

8 Wolf JS, Soble JJ, Ratliff TL, Clayman RV. Ureteral cell cultures I. Characterization and cellular interactions. J Urol 1996; 156: 1198-203

9 Arndt-Jovin DJ, Jovin TM. Analysis and sorting of living cells according to deoxyribonucleic acid content. J Histochem Cytochem 1977; 25: 585-9

10 Shinoka T, Shum-Tim D, Ma PX et al. Creation of viable pulmonary artery autografts through tissue engineering. J Thoracic Cardiovascular Surg 1998; 115: 536-45

11 Yamamoto A, Araki T, Fujimori K et al. $\mathrm{NaCl}$-aided Hoechst 33258 staining method for DNA quantification and its application. Histochem 1989; 92: 65-8

12 Davin-Regli A, Abed Y, Charrel RN, Bollet $\mathrm{C}$, de Micco P. Variations in DNA concentrations significantly affect the reproducibility of RAPD fingerprint patterns. Res Microbiol 1995; 146: 561-8

13 Ellingsen T, Storgaard M, Moller BK et al. Migration of mononuclear cells in the modified Boyden chamber as evaluated by DNA quantification and flow cytometry. Scand J Immunol 2000; 52: 257-63 
14 Parsons CL, Stauffer C, Schmidt JD. Bladder surface glycosaminoglycans. An efficient mechanism of environmental adaptation. Science 1980; 208: 605-7

15 Farndale RW, Buttle D, Barrett AJ. Improved quantitation and discrimination of sulphated glycosaminoglycans by use of dimethylmethylene blue. Biochim Biophys Acta 1986; 883: 173-7

16 Kropp BP, Sawyer BD, Shannon HE etal. Characterization of small intestinal submucosa regenerated canine detrusor: assessment of reinnervation, in vitro compliance, and contractility. J Urol 1996; 156: 599-607

17 Yoo JJ, Meng J, Oberpenning F, Atala A. Bladder augmentation using allogeneic bladder submucosa seeded with cells. Urology 1998; 51 : 221-2
18 Oberpenning F, Meng J, Yoo JJ, Atala A. De novo reconstruction of a functional mammalian urinary bladder by tissue engineering. Nat Biotechnol 1999; 17: 149-55

19 Voytik-Harbin SL, Brightman AO, Kraine MR. al e. Identification of extractable growth factors from small intestinal submucosa. J Cell Biochem 1997; 67: 478-91

20 Zhang Y, Kropp BP, Moore P et al. Coculture of bladder urothelial and smooth muscle cells on small intestinal submucosa: potential applications for tissue engineering technology. J Urol 2000; 164: 928-35

21 Atala A. Autologous cell transplantation for urologic reconstruction. J Urol 1998; 159: $2-3$
22 Wolf JS, Soble JJ, Ratliff TL, Clayman RV. Ureteral cell cultures II. collagen production and response to pharmacologic agents. J Urol 1996; 156: 2067-72

Correspondence: R. Marcovich, MD, Division of Urology, University of Texas Health Sciences Center, 7703 Floyd Curl Drive, MC 7845, San Antonio, TX 78229-3900, USA. e-mail:marcovich@uthscsa.edu

Abbreviations: PGA, polyglycolic acid; ECM, extracellular matrix; UEC, uroepithelial cells; SMC, smooth muscle cells; KSFM, keratinocyte serum-free medium; FBS, fetal bovine serum; TC-P, tissue culture polystyrene; GAG, glycosaminoglycan; SIS small intestinal submucosa. 